\title{
Earth holds comet smash in its sights
}

The largest coordinated observing campaign in astronomy's history is getting under way. More than 60 observatories - and virtually all of the world's big telescopes - are preparing to view the collision between NASA's Deep Impact spacecraft and the unsuspecting comet Tempel 1 on 4 July.

The copper-fortified craft, released from a flyby spacecraft carrying the primary imaging instruments, will smash into the $4 \times 14$-kilometre target at 10 kilometres per second. This will gouge a hole and throw out material from the comet's interior that scientists hope will reveal much about its make-up, and thereby the origins of our Solar System.

Meanwhile, the flyby craft will record the impact from close up with cameras and spectrometers - but only for about 13 minutes. That leaves more distant telescopes on Earth and in space to watch what unfolds as dust, ice and gas continue to spew out from the comet for anywhere between hours and weeks. Earthbased instruments can also view the action at wavelengths unavailable to the spacecraft.

Ground telescopes have already made a major contribution, says Karen Meech of the University of Hawaii, who coordinated the Deep Impact observing campaign. At the start of the mission in 1999, the diameter of the comet's nudeus was known only to within $50 \%$. Years of viewing by Earth-based telescopes (see graphic) mean that it is now known to within a few hundred metres. And ground observers have been monitoring the comet's dust output, partly to gauge the hazard to the spacecraft, and also identifying chemicals in the surrounding coma, so that these can be compared with what flies out of the comet on impact day.

One of Meech's main headaches has been taking into account the different constraints of space- and Earth-based observatories. The Deep Impact team recently decided to push back the spacecraft's arrival time at Tempel 1 by 17 minutes so that the Hubble Space Telescope, whose exact orbit cannot be predicted months in advance, will be in the correct position at impact time. And early on, the team had to choose whether to optimize viewing in Hawaii or Chile, the world's two premier sites for astronomy.
Chile's telescopes had the advantage of being more spread out, which improved the chances of not being rained out on impact day. But timing the impact for viewing in Hawaii meant better coverage from the NASA antennas that communicate with the spacecraft, so Hawaii got the nod.

Meech herself will watch from a control centre on the peak of Mauna Kea in Hawaii that will link astronomers at around $30 \mathrm{global}$ observatories by live video - the first time this has been done for an astronomical event. Other telescopes worldwide will tune in before, during and after the encounter.

Added to that will be hundreds of amateur astronomers, "many of whom are absolutely excellent observers", says Beech, and members of the general public, who should be able to see the brightening cloud of dust spraying from Tempel 1 with binoculars, perhaps even with the naked eye. Viewers from Arizona west to New Zealand will see the impact as it happens, although viewers in Chile won't see the comet until 16 hours later.

Tony Reichhardt

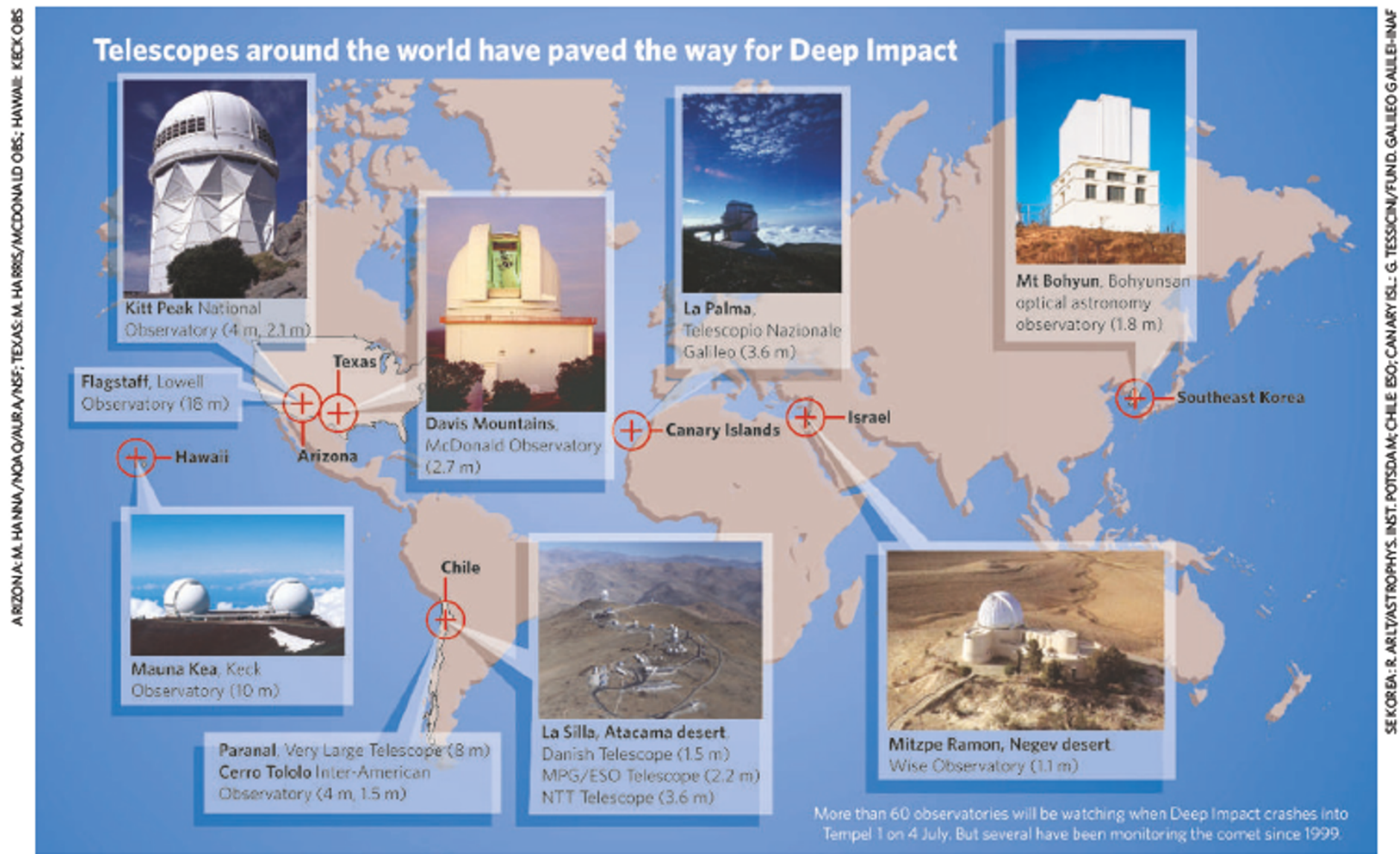

